\title{
Advances on the Probiotic Function of Fermented Lactic Acid Bacteria
}

\author{
Liu Shan-na ${ }^{1,2, ~}$, Li Yun ${ }^{1,2}$, Sun $\mathrm{Xi}^{1,2}$, Wang Na ${ }^{1,2}$, Li Zhi-wen ${ }^{1,2}$ \\ ${ }^{1}$ College of Food Science and Bioengineering, Tianjin Agricultural University, Tianjin, PR China \\ ${ }^{2}$ Tianjin Engineering and Technology Research Center of Agricultural Products Processing, Tianjin, PR China
}

\section{Email address:}

shannaliu@tjau.edu.cn (Liu Shan-na)

\section{To cite this article:}

Liu Shan-na, Li Yun, Sun Xi, Wang Na, Li Zhi-wen. Advances on the Probiotic Function of Fermented Lactic Acid Bacteria. American Journal of Bioscience and Bioengineering. Vol. 3, No. 5, 2015, pp. 118-122. doi: 10.11648/j.bio.20150305.26

\begin{abstract}
Probiotics are current research focus in biotechnology. Their functions in maintaining balance of intestinal microbiota, regulating immune systems and preventing infection of intestinal pathogens have been proved. This article reviewed types and characteristics of probiotics from fermented foods, discussed advances of these probiotics in intestinal tolerance, aggregation and adherence effect, inhibitory activity, immunity and treatment functions, as well as safety. Different probiotic products and their development situations were mentioned. Theoretical references were provided for better utilization of microbial resources.
\end{abstract}

Keywords: Lactic Acid Bacteria, Fermented Food, Probiotic Function

\section{益生性发酵乳酸菌的研究进展}

\author{
刘珊娜 ${ }^{1,2, *}$, 李旳 ${ }^{1,2}$, 孙溪 ${ }^{1,2}$, 王娜 ${ }^{1,2}$, 李志文 ${ }^{1,2}$ \\ '天津农学院, 食品科学与生物工程学院, 天津, 中国 \\ ${ }^{2}$ 天津市农副产品深加工技术工程中心, 天津, 中国
}

\section{邮箱}

shannaliu@tjau. edu. cn（刘珊娜）

摘要: 益生菌是当今生物技术领域的研究热点, 其在维持肠道菌群平衡、调节机体免疫系统及抑制肠道病原菌感染等 方面的作用已得到证实。本文回顾了从发酵食品中分离得到的益生乳酸菌的种类及特点, 探讨了菌株在肠道耐受性、 聚集和粘附作用、抑菌活性、免疫和治疗功能以及安全性等方面的研究进展, 列举了益生乳酸菌产品及开发现状, 为 更好地利用菌种资源提供理论参考。

关键词: 乳酸菌, 发酵食品, 益生功能

\section{1. 引言}

人体肠道的微生物菌群种类多达 500 种，它们在人体 健康中发挥着重要的作用。其中最主要的功能就是通过竞 争环境中的资源防止病原菌的定植, 改善肠道菌群平衡。 国际上将这种口服适当含量对宿主健康有益的活菌称为 益生菌 [1]。益生菌的常见功能包括维持肠道正常菌群,
缓解乳糖不耐症, 降低胆固醇水平, 对抗感染, 抑制癌症, 增强免疫力等。益生菌的基本要求是: 通常被认为是安全 的 (GRAS), 耐酸、耐胃蛋白酶、耐胆盐, 对病原菌有抑制 作用, 能够在肠粘膜定植等。此外, 益生菌应当没有不良 特性如表达毒力因子, 具有有害的生化活性和抗生素抗性 等 $[2]$ 。根据上述特点, 益生菌可应用于人类和动物食品 
中, 提高治疗或保健效果。益生菌食品逐渐成为全球最大 的功能性食品市场, 并且仍在迅猛增长。

乳酸菌 (LAB) 是一类利用糖类发酵产生乳酸的细菌的 通称。乳酸菌发酵食品历史悠久, 种类多样, 为人们广泛 接受。发酵作用不但延长了食品的保存期, 而且改善了食 品的色香味, 增加了营养价值。乳酸菌是人体和动物肠道 内的正常菌群, 它们有长期作为发酵食品成分的安全食用 记录, 能够产生抗菌物质, 阻止肿瘤细胞的生长, 维持肠 道粘膜完整性, 对病原菌起到拮抗作用等 [3]。近年来, 乳酸菌在发酵食品如发酵蔬菜、发酵香肠和发酵乳制品中 的作用及潜在益生功能的研究越来越引起关注, 表现出良 好的应用前景。

\section{2. 益生性发酵乳酸菌的来源}

\section{1. 乳杆菌属 (Lactobacillus) 和双歧杆菌属 (Bifidobacterium)}

乳杆菌属和双歧杆菌属是益生菌的重要来源。乳杆菌 属中常见的益生菌株来自嗜酸乳杆菌 (如鼠李糖乳杆菌、 干酪乳杆菌、副干酪乳杆菌、唾液乳杆菌、格氏乳杆菌、 罗氏乳杆菌和植物乳杆菌), 包括 135 个株和 27 个亚株 [4]。 双歧杆菌属是人体肠道的主要微生物, 能够在新生儿的肠 道中定植并增殖。已用作肠道微生态制剂的有 5 种, 分别 是长双歧杆菌、两歧双歧杆菌、青春双歧杆菌、婴儿双歧 杆菌和短双歧杆菌 [5]。

乳杆菌属是新型益生菌开发领域的研究重点之一, 大 多作为商业或手工生产的发酵剂, 改善发酵食品的风味、 质地和物质转化过程等。研究者在传统发酵酸马奶 [6]、 内蒙古发酵奶制品 [7]、马来西亚当地发酵奶制品 [8]、泡 菜 [9]等食品中都篎选到潜在的益生乳杆菌或双歧杆菌。 于志会等人 $[10]$ 从内蒙古奶豆腐中篮选的植物乳杆菌C88 表现出良好的发酵特性和对金黄色葡萄球菌明显的抑制 作用。Sharaf $i$ 等 [11] 从伊朗传统奶制品中分离得到的面 包乳杆菌KH对酸 $(\mathrm{pH} 2-9)$ 、盐 (1-5\%氯化钠) 和胆盐 $(0.3 \%)$ 具有耐受性, 同时表现出对肠道病原菌 (如大肠杆菌 $0157: H 7)$ 的抗菌活性。

\section{2. 片球菌属 (Pediococcus)}

乳酸片球菌是乳酸菌 23 个属中的一种, 从发酵蔬菜、 酸马奶、发酵香肠等食品中可分离得到。已知乳酸片球菌 可预防一些病原菌如志贺氏菌、沙门氏菌、艰难梭状芽胞 杆菌和大肠杆菌在动物肠道的定植作用, 甚至用于治疗病 毒和寄生虫的感染。Ryu等人 [9]通过体外实验证实泡菜中 分离到的戊糖片球菌MP1符合潜在益生菌功能的要求。 Mandal等人 [12] 从真空包装的发酵肉制品中分离到一株 产细菌素的乳酸片球菌LAB5, 除了耐酸和胆盐外, 还具有 胆固醇清除能力和在宿主上皮细胞的粘附性, 其分泌的细 菌素可抑制特定病原菌的生长。从发酵黄瓜中分离得到的 戊糖片球菌CRAG3除了对酸和胆盐的耐受性外, 还表现出 自聚集、共聚集和体外细胞粘附性 [13]。

\section{3. 明串珠菌属 (Leuconostoc)}

明串珠菌属存在于多种发酵食品中。韩国泡菜中的乳 酸明串珠菌EFEL005对酸和胆盐具有耐受性, 能利用多种 益生元 $[14]$ 。骆超超等人 $[15]$ 从发酵谷物中笁选出赖氨酸 产量高的益生性肠膜明串珠菌ATCC 8293, 动物实验结果 表明, 该菌株具有作为饲料添加剂提高奶牛产奶量和奶品 质量的潜力。巴西的马苏里拉奶酪中分离到的肠膜明串珠 菌肠膜亚种SJRP 55在体外试验中表现出益生活性, 且具有 半乳糖苗酶活性和发酵乳中的存活能力, 可用于开发功能 性食品 [16]。

\section{4. 其它菌属}

乳酸菌中其他种属益生作用的报道较少, 例如发酵面 制品中分离到的海氏肠球菌LD3表现出细胞表面疏水性, 对酸和胆盐耐受性和抗生素敏感性等 [17]。此外从伊朗传 统奶酪中分离得到的粪肠球菌和海氏肠球菌也有相关益 生性的报道[18]。

\section{3. 益生功能的研究}

\section{1. 肠道耐受性的研究}

益生菌只有通过胃和小肠, 到达大肠后粘附在肠道组 织粘膜上, 才能起到定植的作用。Tham等人 [8] 分析了 14 株乳杆菌属和双歧杆菌属菌株, 结果表明, 在 $\mathrm{pH}$ H值 $2-4$ 的 范围内, 大部分菌株都表现出耐受性。当 $\mathrm{pH}$ 值下降到 2 以 下时, 菌株的活力明显下降。Zielińska等人从发酵蔬菜 中分离出 38 株乳杆菌属, 几乎所有菌株可耐受 $\mathrm{pH} 2.5$ 的环 境, 4 株菌株在 $\mathrm{pH} 1.5$ 的环境中能够存活 90 分钟 [19]。相 比而言, 双歧杆菌对高酸性环境更敏感, 在奶制品或胃肠 道环境中存活率很低 $[20]$ 。大多数乳酸菌能够耐受与肠道 相似的胆液环境, 但是对非结合型胆液如胆酸的耐受力差。 双歧杆菌耐胆液的能力要弱于所测定的乳杆菌 [8]。

\section{2. 聚集性和粘附性的研究}

益生菌进入肠道后首先接触的是肠上皮细胞的粘液 层, 粘附的益生菌细胞可以增强在肠道中的停留时间和对 宿主肠道的免疫刺激应答。例如乳杆菌能够形成多细胞聚 合物, 在口腔、人体肠道和阴道中定植。妥彦峰等人 [21] 研究评价了 4 株从传统发酵乳制品中分离到的副干酪乳杆 菌, 其中菌株M5在模拟胃、肠道环境中的耐受性及在肠上 皮细胞上的粘附性较好, 具有潜在的益生功能。此外, 益 生菌与肠道致病菌的凝集可使致病菌更容易从肠道环境 中排出, 有利于宿主抵抗致病菌感染。Nikolic等人 [22] 从手工制作的奶酪中分离到 10 株乳杆菌和 1 株明串珠菌。 利用 $\mathrm{Caco}-2$ 细胞和HT29细胞系对菌株的表面特性进行分 析, 结果表明, 副干酪乳杆菌BGSJ2-8可以与李斯特菌、 大肠杆菌和沙门氏菌发生种间聚集。刘勇等 [23]探索了酸 马奶发酵菌株干酪乳杆菌Zhang细胞表面特性与抗菌能力 之间的关系, 结果证实其对人体肠道致病菌表现出凝集能 力。不同乳酸菌菌株的粘附性存在明显差异, 而粘附机制 目前仍在探索。 


\section{3. 抑菌活性的研究}

定植在肠道内的益生菌通常对肠道致病菌如大肠杆菌、 金黄色葡萄球菌、肺炎克雷伯氏菌、沙门氏菌、李斯特菌 等表现出抑制作用。抑菌机理包括直接产生抑菌物质 (如细 菌素, 过氧化氢, 有机酸等), 产生短链脂肪酸降低环境的 $\mathrm{pH}$ 值, 竞争营养并定植在小肠内壁, 调控免疫应答, 减少 肠腔内细菌的移位等。益生菌的抑菌作用可以是多重的, 并且具有菌株特异性。细菌素主要作用于革兰氏染色阳性 菌, 而革兰氏染色阴性菌通常对有机酸更加敏感。在所测 定的发酵乳酸菌中, 产生的抑菌性代谢产物通常是分泌到 胞外, 扩散到环境中。例如印度高粱发酵食品中分离到的 戊糖乳杆菌和植物乳杆菌发酵液上清具有明显抗生物膜活 性, 有利于环境中病原菌的清除 [24]。抑菌试验主要是在 体外进行, 肠道环境内的抑菌活性目前还未完全阐明。

\section{4. 免疫和治疗功能的研究}

免疫系统功能失调会导致人体过敏和炎症反应的发 生。现有观点认为, 益生菌通过激活巨噬细胞, 增强自然 杀伤细胞活性和免疫球蛋白活性, 提高抗炎症细胞因子表 达水平, 从而改善免疫功能。例如从内蒙古传统发酵酸马 奶中分离的干酪乳杆菌Zhang具有良好的细胞免疫, 体液 免疫和肠粘膜局部免疫等免疫调节功效 [25]。此外, 益生 菌还能预防和辅助治疗高血压; 降低胆固醇, 缓解神经系
统衰老, 预防病理性衰老的发生。研究表明, 从中国西藏 传统开菲尔乳中分离出的植物乳杆菌MA2具有降低体内胆 固醇的功能 [26]。从牦牛酸奶中所篮选的植物乳杆菌能够 显著降低饲喂高脂日粮大鼠血清中胆固醇、甘油三酯、低 密度脂蛋白的含量, 并提高血清瘦素和胰岛素的含量 [27]。 $\mathrm{GaO}$ 等人 [28] 的研究成果也证实清酒乳杆菌C2除了表现出 对肠道环境良好的耐受性和对 $\mathrm{Caco}^{-2}$ 细胞的高粘附率以 外, 还具有较强的清除胆固醇的能力。

\section{5. 安全性研究}

益生菌安全性评价包括病原性、毒性、代谢活性及菌 株内在特性等指标, 通过体外研究、动物试验和人临床研 究来评价菌株的安全性。益生菌可能存在的危害包括: 进 入血液引起人体全身性感染, 产生有害的代谢活性产物, 引发敏感个体的免疫刺激, 长期使用后耐药基因发生转移 等。溶血反应是评价菌株毒力因子的重要方面, 虽然大部 分研究表明益生菌中不存在溶血反应, 但Argyri等人 [29] 也报道了 4 株具有 $\alpha$-溶血能力的戊糖乳杆菌。此外, 抗生 素抗性也是需要考虑的健康风险。徐进等 [30]对中国发酵 乳制品中常用的 137 株益生菌进行安全性评价, 检测到 31 株益生菌对 20 种抗生素具有耐药性, 其中乳杆菌耐药性要 高于双歧杆菌。因此, 新型益生菌菌株在供给消费者之前 必须对潜在的风险因素进行评估。在抗生素、生物胺和酶 活性分析的基础上，进一步进行口服毒性和细菌移位实验。

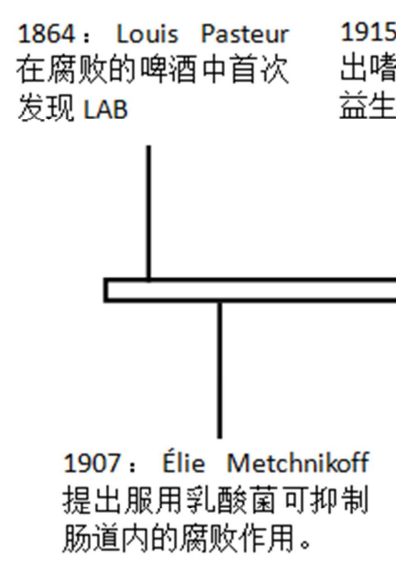

肠道内的腐败作用。

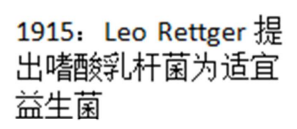

1953: DNA 结 构的确定

\section{9: LAB 和益生} 菌基因组测序
1930: Minoru Shirota 从 人体粪便中分离出植物 乳杆菌并开发出第一款 商业发酵乳饮料, 养乐 多(Yakult)

图1 益生乳酸菌功能特性研究的里程碑 [31]。

\section{4. 应用研究}

益生菌产品种类很多, 包括发酵型酸乳、乳酸菌饮料、 发酵豆制品、发酵型泡菜、发酵肉制品及益生菌制剂等。 其中发酵乳制品是目前最成功、种类最多的一类功能性食 品。例如中国内蒙古地区传统的发酵乳制品开菲尔 (Kefir) 含有大量 “友好的” 微生物菌群, 能够维持和平衡肠道微 生态, 被推荐为康复食品。从传统发酵乳中笁选的益生植 物乳杆菌P8与嗜热链球菌ND03、ND05、ND07复配发酵牛乳, 所得产品酸度适宜, 有良好的粘度且风味较好 [32]。利用 新疆传统奶制品中分离到的植物乳杆菌和干酪乳杆菌制
作的益生菌干酪, 促进小鼠肠道内有益菌显著增殖并具有 明显的调节作用 [33]。将传统奶制品中篮选到的具有理想 益生特性的植物乳杆菌应用于豆乳发酵中, 可以提高豆乳 的粘稠度, 获得良好的口感与风味 [6]。其他类型的发酵 产品包括利用嗜酸乳杆菌发酵发芽小麦饮料 [34], 利用嗜 酸乳杆菌和干酪乳杆菌发酵椰子汁 [35], 利用植物乳杆菌 发酵苹果汁 [36]等。近年来, 益生型乳酸菌还作为肉品发 酵剂生产干发酵香肠, 利用益生菌降低香肠中胆固醇含量, 开发保健型发酵香肠也成为研究中的一个热点。马德功等 人 [37] 从自然发酵香肠中分离出具有益生活性的干酪乳 杆菌和植物乳杆菌, 应用于发酵香肠的生产, 获得品质优 良的产品。 


\section{5. 未来展望}

益生菌概念的提出已有20年的时间，但对这类功能性 菌群的研究仍处于探索阶段。中国发酵乳酸菌资源丰富, 但在益生菌株的基础研究和产品开发上与国际先进水平 相比还存在一定差距。未来的研究中, 益生菌在体内发挥 活性的机理将在分子水平上得到进一步解释, 通过蛋白质 组学、功能基因组学和转录组学的研究阐明益生菌与宿主 间的相互作用。益生菌的应用将更具针对性, 如针对不同 人群, 通过饮食来预防和治疗某些感染性、炎症性或过敏 性疾病。利用叠加效应生产复合益生菌制剂也将为益生菌 制品的开发提供新思路。此外，利用真空冻干技术和微胶 囊包埋技术等对益生菌株进行活性保护也是未来的研究 热点。

\section{致谢}

本文为中国国家自然科学基金青年项目 (31501583) 的阶段性成果之一。

\section{参考文献}

[1] FA0/WHO. Health and nutritional properties of probiotics in food including powder milk with live lactic acid bacteria[R]. Córdoba, Argentina: Food and Agriculture 0rganization of the United Nations, World Health Organization, 2001:4-15.

[2] Clementi F, Aquilanti L. Recent investigations and updated criteria for the assessment of antibiotic resistance in food lactic acid bacteria[J]. Anaerobe, 2011, 17 (6) :394-398.

[3] Lee J, Yun H S, Cho K W, et al. Evaluation of probiotic characteristics of newly isolated Lactobacillus spp. : Immune modulation and longevity[J]. International Journal of Food Microbiology, 2011, 148(2) :80-86.

[4] 杨锡强. 益生菌对免疫功能的影响 $[J]$. 实用儿科临床杂 志, 2010, 25 (9) :623-625.

[5] 高霞, 李卫, 涂世. 益生菌的功效及益生菌乳制品 $[\mathrm{J}]$. 广东 化工, 2009, 36 (5) : 122-123.

[6] Bao Y, Zhang Y, Li H, et al. In vitro screen of Lactobacillus plantarum as probiotic bacteria and their fermented characteristics in soymilk[J]. Annals of Microbiology, 2012, 62 (3) :1311-1320.

[7] 间丽雅. 六株益生菌在豆乳中的发酵特性研究 [D]. 呼和浩 特: 内蒙古农业大学, 2010:1-2.

[8] Tham C S C, Peh K K, Bhat R, et al. Probiotic properties of bifidobacteria and lactobacilli isolated from local dairy products[J]. Annals of Microbiology, 2012, $62(3): 1079-1087$.
[9] Ryu E H, Chang H C. In vitro study of potentially probiotic lactic acid bacteria strains isolated from kimchi[J]. Annals of Microbiology, 2013, 63 (4) :1387-1395.

[10] 于志会, 侯聚敏, 李达, 等. 植物乳杆菌C88发酵特性研究 [J]. 中国乳品工业, 2012, 40 (3) : 24-27.

[11] Sharafi H, Derakhshan V, Paknejad M, et al. Lactobacillus crustorum KH: Novel prospective probiotic strain isolated from Iranian traditional dairy products[J]. Applied Biochemistry \& Biotechnology, 2014, 175(4):2178-2194.

[12] Mandal V, Sen S K, Mandal N C. Effect of prebiotics on bacteriocin production and cholesterol lowering activity of Pediococcus acidilactici LAB 5[J]. World Journal Microbiology and Biotechnology, 2009, 25 (10) : 1837-1847.

[13] Shukla R, Goyal A. Probiotic Potential of Pediococcus pentosaceus CRAG3: A new isolate from fermented cucumber $[J]$. Probiotics \& Antimicrobial Proteins, 2014, 6 (1) :11-21.

[14] Jin S M, Choi H S, Shin S Y, et al. Genome sequence analysis of potential probiotic strain Leuconostoc lactis EFEL005 isolated from kimchi[J]. Journal of Microbiology, 2015, 53(5) :337-342.

[15] 骆超超. 发酵谷物中产赖氨酸益生菌的篮选及其应用 $[D]$. 沈阳:东北农业大学, 2010:14-19.

[16] Paula A T D, Jeronymo-Ceneviva A B, Silva L F, et al. Leuconostoc mesenteroides SJRP55: A potential probiotic strain isolated from Brazilian water buffalo Mozzarella cheese[J]. Annals of Microbiology, 2015, 65:899-910.

[17] Gupta A, Tiwari S K. Probiotic potential of bacteriocin-producing Enterococcus hirae strain LD3 isolated from dosa batter [J]. Annals of Microbiology, 2015, DOI 10. 1007/s13213-015-1075-4.

[18] Ghahremani E, Mardani M, Rezapour S. Phenotypic and genotypic characterization of lactic acid bacteria from traditional cheese in Khorramabad city of Iran with probiotic potential[J]. Applied Biochemistry Biotechnology, 2015, 175:2516-2527.

[19] Zielińska D, Rzepkowska Anna, Radawska Anna, et al. In vitro screening of selected probiotic properties of Lactobacillus strains isolated from traditional fermented cabbage and cucumber $[J]$. Current Microbiology, 2014, $70(2)$ : 183-194.

[20] Doleyres Y, Lacroix C. Technologies with free and immobilized cells for probiotic bifidobacteria production and protection[J]. International Dairy Journal, 2005, 15 (10):973-988. 
[21] 妥彦峰, 张伟钦, 张兰威, 等. Lactobacillus菌株的益生功 能评价 [J]. 现代食品科技, 2012, 28(8):906-910.

[22] Nikolic M, Jovcic B, Kojic M, et al. Surface properties of Lactobacillus and Leuconostoc isolates from homemade cheeses showing auto-aggregation ability[J]. European Food Research and Technology, 2010, 231 (6) : 925-931.

[23] 刘勇, 张勇, 包艳, 等. 4株益生菌的表面特性及抑制致病菌 作用研究 [J]. 中国食品学报, 2010, 10 (2) :28-34.

[24] Rao K P, Chennappa G, Suraj U, et al. Probiotic potential of Lactobacillus strains isolated from sorghum-based traditional fermented food[J]. Probiotics \& Antimicrobial Proteins, 2015, 7 (2) : 146-156.

[25] 刘文俊, 乌日娜, 张和平. 益生菌L. casei Zhang的多项分类 鉴定 [J]. 中国乳品工业, 2009, 37 (2) : 14-18.

[26] Wang Y, Xu N, Xi A, et al. Effects of Lactobacillus plantarum MA2 isolated from Tibet kefir on lipid metabolism and intestinal microflora of rats fed on high-cholesterol diet[J]. Applied Microbiology and Biotechnology, 2009, 84(2):341-347.

[27] 丁武蓉. 青藏高原传统发酵牦牛奶中乳酸菌多样性及其益 生功能研究 [D]. 兰州: 兰州大学, 2014:60-62.

[28] Gao Y, Li D, Liu S, et al. Probiotics potential of $L$. sake $\mathrm{C} 2$ isolated from traditional Chinese fermented cabbage $[\mathrm{J}]$. European Food Research and Technology, 2012, $234(1): 45-51$.

[29] Argyri A A, Zoumpopoulou G, Karatzas K A G, et al. Selection of potential probiotic lactic acid bacteria from fermented olives by in vitro tests $[\mathrm{J}]$. Food microbiology, 2013, 33 (2) :282-291.

[30] 徐进, 严卫星, 杨宝兰, 等. 中国发酵乳制品益生菌菌种的安 全性评估 [J]. 卫生研究, 2008, 37 (2) : 193-195.

[31] Johnson B R, Klaenhammer T R. Impact of genomics on the field of probiotic research: historical perspectives to modern paradigms $[J]$. Antonie Van Leeuwenhoek, 2014, 106(1):141-156.

[32] 何秋雯, 王水泉, 鲍雅静, 等. 益生菌Lactobacillus plantarum P8与Streptococcus thermophilus混合发酵对 发酵乳品质的影 [J]. 中国乳品工业, 2012, 40 (10) : 8-13.

[33] 冯思明, 王琴, 时玉中, 等. 益生菌干酪对小白鼠肠道微生态 区的影响 [J]. 食品与发酵科技, 2012, 48 (4) : 42-44.

[34] Sharma M, Mridula D, Gupta R K. Development of sprouted wheat based probiotic beverage[J]. Journal of Food Science and Technology, 2014, 51 (12) :3926-3933.

[35] Lee P, Boo C X, Liu S. Fermentation of coconut water by probiotic strains Lactobacillus acidophilus L10 and Lactobacillus casei L26[J]. Annals of Microbiology, 2013, 63:1441-1450.

[36] Dimitrovski D, Velickova E, Langerholc T, et al. Apple juice as a medium for fermentation by the probiotic Lactobacillus plantarum PCS 26 strain[J]. Annals of Microbiology, 2015, D0I 10.1007/s13213-015-1056-7.

[37] 马德功, 发酵香肠中乳酸菌的分离、篮选及其应用 $[D]$. 济南: 山东轻工业学院, 2008:40-49. 